\title{
QUOTIENT OF LAPLACE AND GUMBEL RANDOM VARIABLES
}

\author{
SARALEES NADARAJAH
}

Received 5 July 2005; Revised 13 December 2005; Accepted 13 March 2006

The Gumbel and the Laplace distributions are perhaps two of the most applied distributions in engineering. Motivated by engineering issues, the exact distribution of the quotient $|X / Y|$ is derived when $X$ and $Y$ are independent Gumbel and Laplace random variables. Tabulations of the associated percentage points and a computer program for generating them are also given.

Copyright (C) 2006 Saralees Nadarajah. This is an open access article distributed under the Creative Commons Attribution License, which permits unrestricted use, distribution, and reproduction in any medium, provided the original work is properly cited.

\section{Introduction}

The Gumbel and the Laplace distributions are perhaps two of the most applied distributions in engineering. The Gumbel distribution is also known as the extreme value distribution of type I. Some of its recent application areas in engineering include flood frequency analysis, network engineering, nuclear engineering, offshore engineering, riskbased engineering, space engineering, software reliability engineering, structural engineering, and wind engineering. A recent book by Kotz and Nadarajah [4], which describes this distribution, lists over fifty applications ranging from accelerated life testing to earthquakes, floods, horse racing, rainfall, queues in supermarkets, sea currents, and wind speeds (to mention just a few).

The Laplace distribution has found applications in a variety of engineering areas that range from image and speech recognition to ocean engineering. They are rapidly becoming distributions of first choice whenever "something" with heavier than Gaussian tails is observed in the data.

Since Gumbel and Laplace distributions are popular in engineering, there are many real situations where measurements could be modeled by these distributions. Some examples are:

(1) in communication theory, $X$ and $Y$ could represent the random noise corresponding to two signals; 
(2) in ocean engineering, $X$ and $Y$ could represent distributions of navigation errors;

(3) in image and speech recognition, $X$ and $Y$ could represent "input" distributions;

(4) in chemical engineering, $X$ and $Y$ could represent the remission times of two chemicals when they are administered to two kinds of mechanical systems;

(5) in civil engineering, $X$ and $Y$ could represent future observations on the strength of an engineering design (e.g., the strength of a bridge);

(6) in hydrology, $X$ and $Y$ could represent the extreme rainfall at two stations.

In each of the examples above, it will be of interest to study the distribution of the quotient $|X / Y|$. For example, in communication theory, $|X / Y|$ could represent the relative strength of the two different signals. In ocean engineering, $|X / Y|$ could represent the relative safety of navigation. In mechanical engineering, $|X / Y|$ could represent the relative effectiveness of the two chemicals. In civil engineering, $|X / Y|$ could represent some measure of reliability of the engineering design. In hydrology, $|X / Y|$ could represent the relative extremity of rainfall at the two stations.

The distribution of the quotient $X / Y$ has been studied by several authors especially when $X$ and $Y$ are independent random variables and come from the same family. For instance, see Marsaglia [5] and Korhonen and Narula [3] for normal family, Press [7] for Student's $t$ family, Basu and Lochner [1] for Weibull family, Shcolnick [9] for stable family, Hawkins and Han [2] for noncentral chi-squared family, Provost [8] for gamma family, and Pham-Gia [6] for beta family.

However, there is relatively little work of this kind when $X$ and $Y$ belong to different families. In this note, we study the exact distribution of $|X / Y|$ when $X$ and $Y$ are independent Gumbel and Laplace random variables with pdf's

$$
\begin{gathered}
f_{X}(x)=\exp \left(-\frac{x-\mu}{\sigma}\right) \exp \left\{-\exp \left(-\frac{x-\mu}{\sigma}\right)\right\}, \\
f_{Y}(y)=\frac{\lambda}{2} \exp \{-\lambda|y-\theta|\},
\end{gathered}
$$

respectively, for $-\infty<x<\infty,-\infty<y<\infty,-\infty<\mu<\infty,-\infty<\theta<\infty, \sigma>0$, and $\lambda>0$. Tabulations of the associated percentage points and a computer program for generating them are also provided. The calculations involve several special functions, including the incomplete gamma function defined by

$$
\gamma(a, x)=\int_{0}^{x} t^{a-1} \exp (-t) d t
$$

and the complementary incomplete gamma function defined by

$$
\Gamma(a, x)=\int_{x}^{\infty} t^{a-1} \exp (-t) d t
$$


The properties of these special functions can be found in Prudnikov et al. (1986) and Gradshteyn and Ryzhik (2000).

\section{Cumulative distribution function}

Theorem 2.1 derives explicit expressions for the cdf of $|X / Y|$ in terms of the incomplete gamma functions.

Theorem 2.1. Suppose $X$ and $Y$ are distributed according to (1.1) and (1.2), respectively. The cdf of $Z=|X / Y|$ can be expressed as one of the following:

(1) if $\theta<0$, then

$$
\begin{aligned}
F(z)=\frac{\lambda \sigma}{2 z}\left[\frac{1}{a b} \gamma\left(\frac{\lambda \sigma}{z}, c d\right)+a b\left\{\Gamma\left(-\frac{\lambda \sigma}{z}, c d\right)-\Gamma\left(-\frac{\lambda \sigma}{z}, c\right)\right\}+\frac{a}{b} \gamma\left(\frac{\lambda \sigma}{z}, c\right)\right. \\
\left.\quad-\frac{b}{a} \Gamma\left(-\frac{\lambda \sigma}{z}, \frac{c}{d}\right)-\frac{a}{b}\left\{\gamma\left(\frac{\lambda \sigma}{z}, \frac{c}{d}\right)-\gamma\left(\frac{\lambda \sigma}{z}, c\right)\right\}-a b \Gamma\left(-\frac{\lambda \sigma}{z}, c\right)\right]
\end{aligned}
$$

(2) if $\theta=0$, then

$$
F(z)=\frac{\lambda \sigma}{z}\left[\frac{1}{b} \gamma\left(\frac{\lambda \sigma}{z}, c\right)-b \Gamma\left(-\frac{\lambda \sigma}{z}, c\right)\right]
$$

(3) if $\theta<0$, then

$$
\begin{aligned}
F(z)=\frac{\lambda \sigma}{2 z}\left[\frac{a}{b} \gamma\left(\frac{\lambda \sigma}{z}, \frac{c}{d}\right)+\frac{b}{a}\left\{\Gamma\left(-\frac{\lambda \sigma}{z}, \frac{c}{d}\right)-\Gamma\left(-\frac{\lambda \sigma}{z}, c\right)\right\}+\frac{1}{a b} \gamma\left(\frac{\lambda \sigma}{z}, c\right)\right. \\
\left.-a b \Gamma\left(-\frac{\lambda \sigma}{z}, c d\right)-\frac{1}{a b}\left\{\gamma\left(\frac{\lambda \sigma}{z}, c d\right)-\gamma\left(\frac{\lambda \sigma}{z}, c\right)\right\}-\frac{b}{a} \Gamma\left(-\frac{\lambda \sigma}{z}, c\right)\right]
\end{aligned}
$$

for $z>0$, where $a=\exp (\lambda \theta), b=\exp (\lambda \mu / z), c=\exp (\mu / \sigma)$, and $d=\exp (\theta z / \sigma)$.

Proof. One can write

$$
\begin{aligned}
\operatorname{Pr}(|X / Y| \leq z)= & \int_{-\infty}^{\infty}\left\{F_{X}(|y| z)-F_{X}(-|y| z)\right\} f_{Y}(y) d y \\
= & \frac{\lambda}{2}\left[\int_{-\infty}^{\theta} \exp \left\{\lambda(y-\theta)-\exp \left(\frac{\mu+y z}{\sigma}\right)\right\} d y\right. \\
& +\int_{\theta}^{0} \exp \left\{\lambda(\theta-y)-\exp \left(\frac{\mu+y z}{\sigma}\right)\right\} d y \\
& +\int_{0}^{\infty} \exp \left\{\lambda(\theta-y)-\exp \left(\frac{\mu-y z}{\sigma}\right)\right\} d y
\end{aligned}
$$


4 Quotient of Laplace and Gumbel random variables

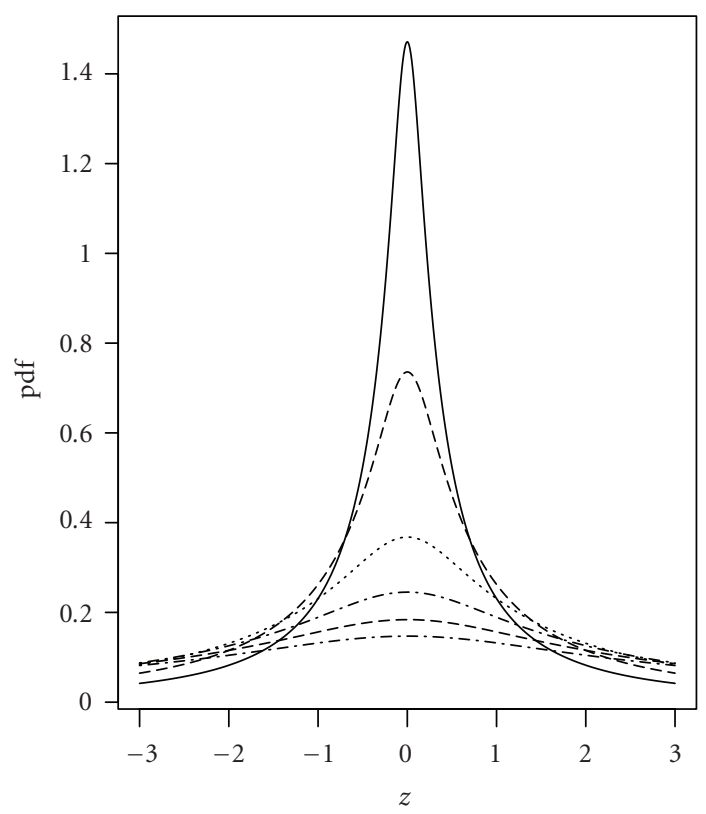

Figure 2.1. Plots of the pdf of (2.5) for $\lambda \sigma=0.5,1,2,3,4,5$. The curves from the top to the bottom correspond to increasing values of $\lambda \sigma$.

$$
\begin{aligned}
& -\int_{-\infty}^{\theta} \exp \left\{\lambda(y-\theta)-\exp \left(\frac{\mu-y z}{\sigma}\right)\right\} d y \\
& -\int_{\theta}^{0} \exp \left\{\lambda(\theta-y)-\exp \left(\frac{\mu-y z}{\sigma}\right)\right\} d y \\
& \left.-\int_{0}^{\infty} \exp \left\{\lambda(\theta-y)-\exp \left(\frac{\mu+y z}{\sigma}\right)\right\} d y\right] \\
\frac{\lambda \sigma}{2 z}[ & \frac{1}{a b} \int_{0}^{c d} v^{\lambda \sigma / z-1} \exp (-v) d v+a b \int_{c d}^{c} v^{-\lambda \sigma / z-1} \exp (-v) d v \\
& +\frac{a}{b} \int_{0}^{c} u^{\lambda \sigma / z-1} \exp (-u) d u-\frac{b}{a} \int_{c / d}^{\infty} u^{-\lambda \sigma / z-1} \exp (-u) d u \\
& \left.-\frac{a}{b} \int_{c}^{c / d} u^{\lambda \sigma / z-1} \exp (-u) d u-a b \int_{c}^{\infty} v^{-\lambda \sigma / z-1} \exp (-v) d v\right],
\end{aligned}
$$

where the last step follows by substituting $u=\exp \{(\mu-y z) / \sigma\}$ and $v=\exp \{(\mu+y z) / \sigma\}$. The result in (2.1) follows from (2.4) by using the definitions of the incomplete gamma 
functions defined in Section 1. Setting $\theta=0$ gives the result in (2.2). Replace $\theta$ by $-\theta$ in (2.1) to obtain the result in (2.3).

Consider the standard Gumbel and Laplace distributions with $\mu=0$ and $\theta=0$. In this case, (2.2) reduces to the simpler form

$$
F(z)=\frac{\lambda \sigma}{z}\left\{\gamma\left(\frac{\lambda \sigma}{z}, 1\right)-\Gamma\left(-\frac{\lambda \sigma}{z}, 1\right)\right\}
$$

Note that (2.5) depends only on $\lambda \sigma$, the product of the scale parameters. Furthermore, (2.5) reduces to degenerate distributions in the limiting cases $\lambda \sigma \rightarrow 0$ and $\lambda \sigma \rightarrow \infty$.

Figure 2.1 above illustrates possible shapes of the pdf of (2.5) for a range of values of $\lambda \theta$. The effect of the parameter is evident. The parameters $\mu$ and $\theta$ simply control the location of the pdf of $Z=|X / Y|$.

\section{Percentiles}

In this section, we provide tabulations of percentage points $z_{p}$ associated with the cdf (2.5) of $Z=|X / Y|$. These values are obtained by numerically solving the equation

$$
\frac{\lambda \sigma}{z_{p}}\left\{\gamma\left(\frac{\lambda \sigma}{z_{p}}, 1\right)-\Gamma\left(-\frac{\lambda \sigma}{z_{p}}, 1\right)\right\}=p .
$$

Evidently, this involves computation of the incomplete gamma functions and routines for this are widely available. We used the function $\operatorname{GAMMA}(\cdot, \cdot)$ in the algebraic manipulation package, MAPLE. Table 3.1 provides the numerical values of $z_{p}$ for $\lambda \sigma=0.1,0.2, \ldots, 5$.

We hope these numbers will be of use to the practitioners mentioned in the introduction. Similar tabulations could be easily derived for other values of $\lambda \sigma$ and $p$ by using the GAMMA $(\cdot)$ function in MAPLE. A sample program is shown in the appendix below.

\section{Appendix}

The following program in MAPLE can be used to generate tables similar to that presented in Section 3.

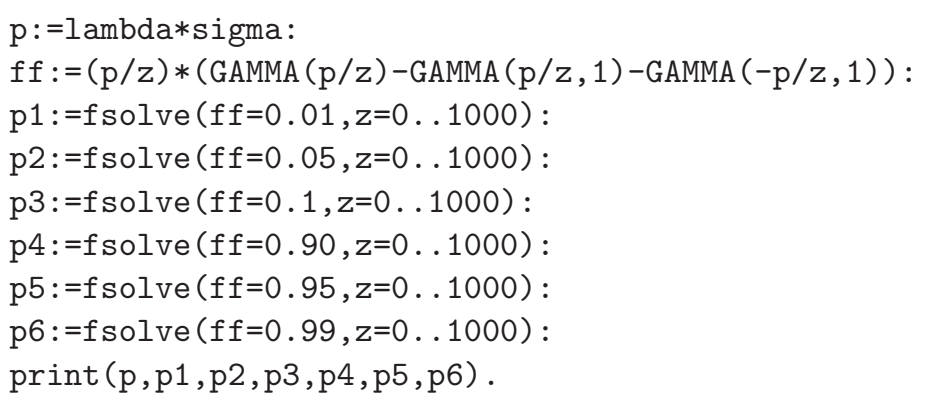


6 Quotient of Laplace and Gumbel random variables

Table 3.1. Percentage points of $Z=|X / Y|$.

\begin{tabular}{|c|c|c|c|c|c|c|}
\hline$\lambda \sigma$ & $p=0.01$ & $p=0.05$ & $p=0.1$ & $p=0.9$ & $p=0.95$ & $p=0.99$ \\
\hline 0.1 & 0.001351115 & 0.006812125 & 0.01381703 & 0.9186777 & 1.942657 & 10.00132 \\
\hline 0.2 & 0.002712925 & 0.01359741 & 0.02769946 & 1.832344 & 3.841862 & 20.18182 \\
\hline 0.3 & 0.004148245 & 0.02036646 & 0.04128311 & 2.755540 & 5.816555 & 30.3209 \\
\hline 0.4 & 0.005349738 & 0.02722697 & 0.05517113 & 3.673644 & 7.737383 & 40.45507 \\
\hline 0.5 & 0.00674141 & 0.0341019 & 0.0692158 & 4.597857 & 9.638007 & 49.83905 \\
\hline 0.6 & 0.008188162 & 0.04105924 & 0.08344926 & 5.515774 & 11.61478 & 60.44436 \\
\hline 0.7 & 0.009696907 & 0.04762376 & 0.0966465 & 6.3995 & 13.44198 & 70.90835 \\
\hline 0.8 & 0.01068737 & 0.05465819 & 0.1110102 & 7.373572 & 15.57044 & 81.81201 \\
\hline 0.9 & 0.01209260 & 0.06075592 & 0.1239814 & 8.212888 & 17.25587 & 89.18965 \\
\hline 1 & 0.01367985 & 0.06800081 & 0.1383829 & 9.189068 & 19.33069 & 100.2222 \\
\hline 1.1 & 0.01486944 & 0.07500787 & 0.1519422 & 10.15826 & 21.43559 & 111.9211 \\
\hline 1.2 & 0.01600875 & 0.08152697 & 0.1657749 & 11.04029 & 23.12398 & 120.1613 \\
\hline 1.3 & 0.0175391 & 0.08907592 & 0.1799106 & 11.99019 & 25.08107 & 129.6253 \\
\hline 1.4 & 0.01926416 & 0.09644032 & 0.1949094 & 12.90918 & 27.32613 & 142.1615 \\
\hline 1.5 & 0.02046504 & 0.1026064 & 0.2077930 & 13.74593 & 29.08487 & 150.0171 \\
\hline 1.6 & 0.02182045 & 0.1097083 & 0.2217979 & 14.71370 & 30.85967 & 160.568 \\
\hline 1.7 & 0.02300527 & 0.1157000 & 0.2350247 & 15.66953 & 32.95361 & 172.6839 \\
\hline 1.8 & 0.02429262 & 0.1222268 & 0.2482944 & 16.48039 & 34.86024 & 175.9620 \\
\hline 1.9 & 0.02525058 & 0.1286303 & 0.2629522 & 17.48526 & 36.72677 & 191.7719 \\
\hline 2 & 0.02725426 & 0.1356990 & 0.275934 & 18.44431 & 38.85844 & 205.2303 \\
\hline 2.1 & 0.02857707 & 0.1440879 & 0.2913955 & 19.22595 & 40.47796 & 212.9673 \\
\hline 2.2 & 0.03012728 & 0.1499321 & 0.3045255 & 20.19328 & 42.291 & 219.4435 \\
\hline 2.3 & 0.03129156 & 0.1566246 & 0.3178748 & 21.20539 & 44.73074 & 235.1875 \\
\hline 2.4 & 0.03281368 & 0.1638796 & 0.3324096 & 22.00995 & 46.21768 & 239.447 \\
\hline 2.5 & 0.03387920 & 0.1712344 & 0.3475101 & 23.07892 & 48.56283 & 250.0943 \\
\hline 2.6 & 0.03593847 & 0.1789796 & 0.3625134 & 24.01946 & 50.68632 & 264.2627 \\
\hline 2.7 & 0.03653574 & 0.1842649 & 0.3737424 & 24.89275 & 52.17294 & 269.2083 \\
\hline 2.8 & 0.03844623 & 0.1916820 & 0.3879259 & 25.66422 & 54.3857 & 284.8752 \\
\hline 2.9 & 0.03888073 & 0.1967611 & 0.4003512 & 26.68252 & 56.19697 & 297.0643 \\
\hline 3 & 0.04132923 & 0.2046606 & 0.415163 & 27.57299 & 58.0705 & 303.4964 \\
\hline 3.1 & 0.04208519 & 0.21221 & 0.4283762 & 28.48251 & 59.96408 & 313.4921 \\
\hline 3.2 & 0.04330353 & 0.2184272 & 0.4438443 & 29.36639 & 61.54506 & 315.8305 \\
\hline 3.3 & 0.0444831 & 0.2250572 & 0.4574535 & 30.28838 & 63.70593 & 334.9964 \\
\hline 3.4 & 0.04575357 & 0.2309297 & 0.4685282 & 31.27244 & 66.05638 & 342.3006 \\
\hline 3.5 & 0.04712695 & 0.2398521 & 0.4842837 & 32.50126 & 68.54356 & 357.3495 \\
\hline 3.6 & 0.04930681 & 0.2469442 & 0.5021143 & 33.12675 & 69.79813 & 361.953 \\
\hline 3.7 & 0.05030483 & 0.2544659 & 0.5145381 & 34.06142 & 71.63831 & 372.2156 \\
\hline 3.8 & 0.05092874 & & & & 73.13104 & 385.4243 \\
\hline 3.9 & 0.0530573 & 0.2653743 & 0.5400184 & 35.68201 & 74.82779 & 391.4862 \\
\hline 4 & 0.05417395 & 0.2727553 & 0.5535613 & 36.68836 & 76.95862 & 406.8885 \\
\hline 4.1 & 0.05503099 & 0.280475 & 0.5696706 & 37.69073 & 79.4004 & 411.0571 \\
\hline 4.2 & 0.05783188 & 0.2876157 & 0.5805482 & 38.61597 & 81.48548 & 426.8407 \\
\hline 4.3 & 0.05874114 & 0.2939004 & 0.5946736 & 39.53981 & 83.52469 & 437.0593 \\
\hline 4.4 & 0.05921993 & 0.3006934 & 0.6121295 & 40.38349 & 85.50906 & 449.4799 \\
\hline 4.5 & 0.06238164 & 0.3073551 & 0.6228517 & 41.57249 & 87.40623 & 454.776 \\
\hline 4.6 & 0.06285294 & 0.3153175 & 0.638604 & 42.31586 & 88.88288 & 461.6626 \\
\hline 4.7 & 0.06354897 & 0.3213952 & 0.651258 & 43.36174 & 91.12971 & 467.8765 \\
\hline 4.8 & 0.0654103 & 0.3283733 & 0.66615 & 44.29262 & 92.92313 & 478.6359 \\
\hline 4.9 & 0.0677629 & 0.3356406 & 0.6803458 & 44.89927 & 94.93208 & 492.1779 \\
\hline 5 & 0.06880682 & 0.3424891 & 0.6953104 & 46.10605 & 96.7116 & 497.5622 \\
\hline
\end{tabular}




\section{Acknowledgments}

The author would like to thank the referee and the editor for carefully reading the paper and for their help in improving the paper.

\section{References}

[1] A. P. Basu and R. H. Lochner, On the distribution of the ratio of two random variables having generalized life distributions, Technometrics 13 (1971), 281-287.

[2] D. L. Hawkins and C.-P. Han, Bivariate distributions of some ratios of independent noncentral chi-square random variables, Communications in Statistics. A. Theory and Methods 15 (1986), no. $1,261-277$.

[3] P. J. Korhonen and S. C. Narula, The probability distribution of the ratio of the absolute values of two normal variables, Journal of Statistical Computation and Simulation 33 (1989), no. 3, $173-182$.

[4] S. Kotz and S. Nadarajah, Extreme Value Distributions: Theory and Applications, Imperial College Press, London, 2000.

[5] G. Marsaglia, Ratios of normal variables and ratios of sums of uniform variables, Journal of the American Statistical Association 60 (1965), 193-204.

[6] T. Pham-Gia, Distributions of the ratios of independent beta variables and applications, Communications in Statistics. Theory and Methods 29 (2000), no. 12, 2693-2715.

[7] S. J. Press, The t ratio distribution, Journal of the American Statistical Association 64 (1969), 242-252.

[8] S. B. Provost, On the distribution of the ratio of powers of sums of gamma random variables, Pakistan Journal of Statistics 5 (1989), no. 2, 157-174.

[9] S. M. Shcolnick, On the ratio of independent stable random variables, Stability Problems for Stochastic Models (Uzhgorod, 1984), Lecture Notes in Math., vol. 1155, Springer, Berlin, 1985, pp. 349-354.

Saralees Nadarajah: Department of Statistics, University of Nebraska, Lincoln, NE 68583, USA

E-mail address: snadaraj@unlserve.unl.edu 


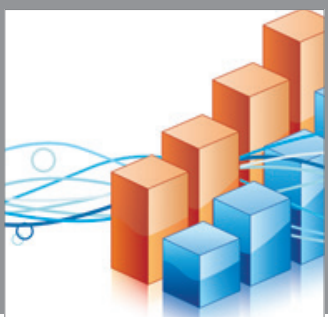

Advances in

Operations Research

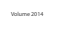

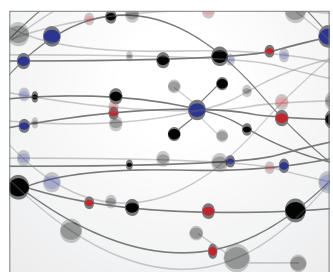

\section{The Scientific} World Journal
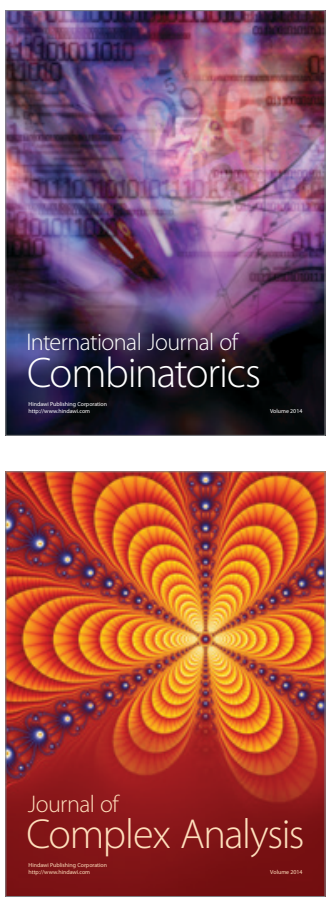

International Journal of

Mathematics and

Mathematical

Sciences
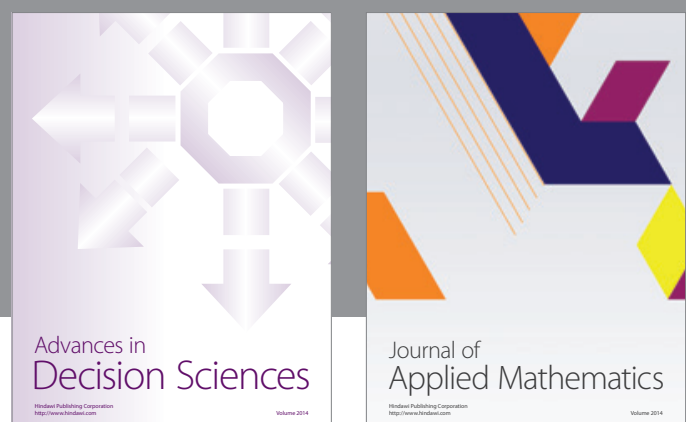

Journal of

Applied Mathematics
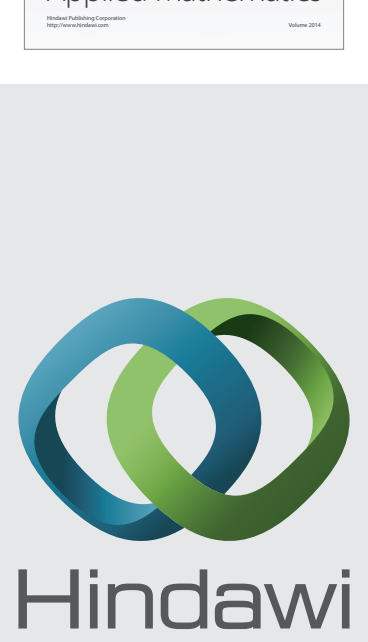

Submit your manuscripts at http://www.hindawi.com
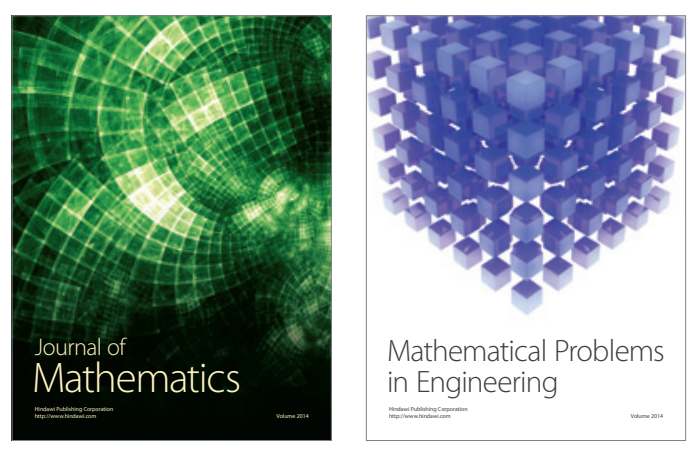

Mathematical Problems in Engineering
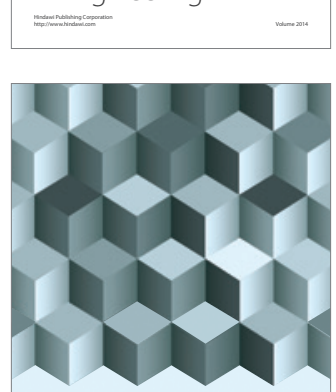

Journal of

Function Spaces
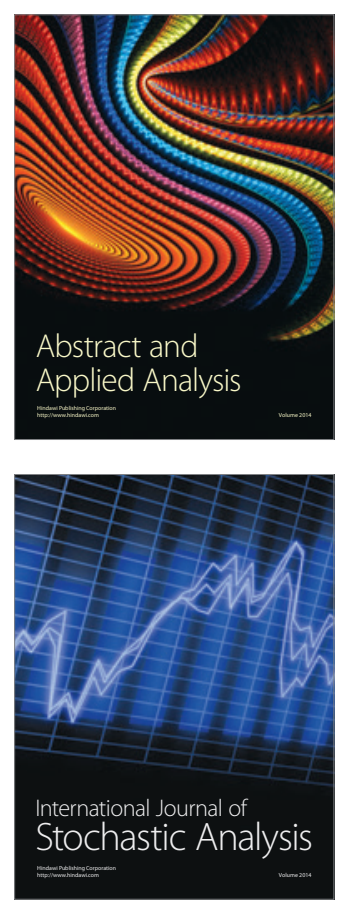

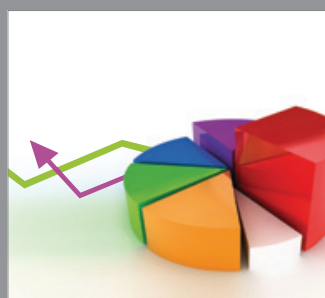

ournal of

Probability and Statistics

Promensencen
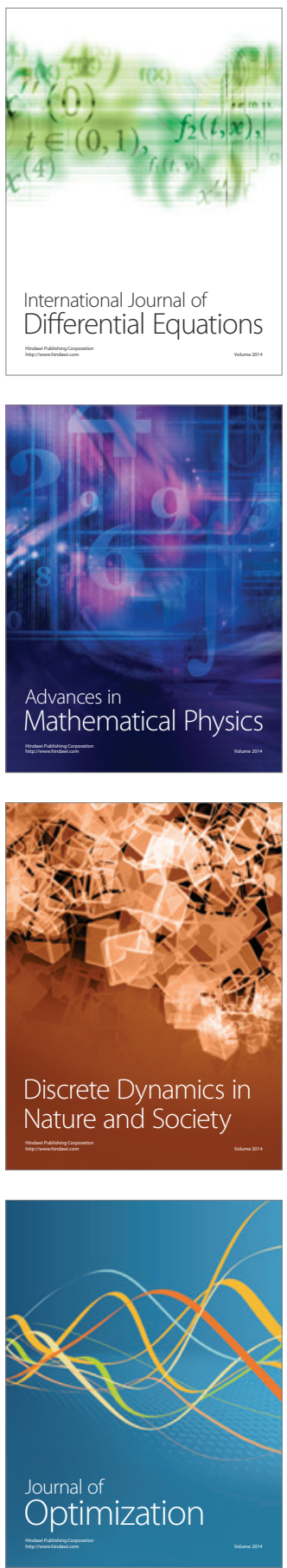